\title{
WASP-52b, WASP-58b, WASP-59b, and WASP-60b: Four new transiting close-in giant planets ${ }^{\star}$
}

\author{
G. Hébrard ${ }^{1,2}$, A. Collier Cameron ${ }^{3}$, D. J. A. Brown ${ }^{3}$, R. F. Díaz ${ }^{4,1}$, F. Faedi ${ }^{13}$, B. Smalley ${ }^{5}$, D. R. Anderson ${ }^{5}$,
} D. Armstrong ${ }^{13}$, S. C. C. Barros ${ }^{4}$, J. Bento ${ }^{12}$, F. Bouchy ${ }^{1,2}$, A. P. Doyle ${ }^{5}$, B. Enoch ${ }^{3}$, Y. Gómez Maqueo Chew ${ }^{13,14}$, É. M. Hébrard ${ }^{2}$, C. Hellier ${ }^{5}$, M. Lendl ${ }^{6}$, T. A. Lister ${ }^{11}$, P. F. L. Maxted ${ }^{5}$, J. McCormac ${ }^{8}$, C. Moutou ${ }^{4}$, D. Pollacco ${ }^{13}$, D. Queloz ${ }^{6}$, A. Santerne ${ }^{4,2}$, I. Skillen ${ }^{8}$, J. Southworth ${ }^{5}$, J. Tregloan-Reed ${ }^{5}$, A. H. M. J. Triaud ${ }^{6}$, S. Udry $^{6}$, M. Vanhuysse ${ }^{10}$, C. A. Watson ${ }^{7}$, R. G. West ${ }^{9}$, and P. J. Wheatley ${ }^{13}$

1 Institut d'Astrophysique de Paris, UMR 7095 CNRS, Université Pierre \& Marie Curie, 98bis boulevard Arago, 75014 Paris, France e-mail: hebrard@iap.fr

2 Observatoire de Haute-Provence, CNRS/OAMP, 04870 Saint-Michel-l'Observatoire, France

3 School of Physics and Astronomy, University of St Andrews, St Andrews, Fife KY16 9SS, UK

${ }^{4}$ Laboratoire d'Astrophysique de Marseille, Univ. de Provence, CNRS (UMR 6110), 38 r. F. Joliot Curie, 13388 Marseille Cedex 13, France

5 Astrophysics Group, Keele University, Staffordshire, ST5 5BG, UK

${ }^{6}$ Observatoire de Genève, Université de Genève, 51 chemin des Maillettes, 1290 Sauverny, Switzerland

7 Astrophysics Research Centre, School of Mathematics and Physics, Queens University Belfast, University Road, Belfast BT7 1NN, UK

8 Isaac Newton Group of Telescopes, Apartado de Correos 321, 38700 Santa Cruz de Palma, Spain

9 Department of Physics and Astronomy, University of Leicester, Leicester, LE1 7RH, UK

10 OverSky, 47 allée des Palanques, BP 12, 33127 Saint-Jean d'Illac, France

11 Las Cumbres Observatory, 6740 Cortona Drive Suite 102, Goleta, CA 93117, USA

12 The Open University, Walton Hall, Milton Keynes, MK7 6AA, UK

13 Department of Physics, University of Warwick, Gibbet Hill Road, Coventry CV4 7AL, UK

14 Department of Physics and Astronomy, Vanderbilt University, 6301 Stevenson Center, Nashville, TN 37235, USA

Received 11 September 2012 / Accepted 5 November 2012

\section{ABSTRACT}

\begin{abstract}
We present the discovery of four new transiting hot Jupiters, detected mainly from SuperWASP-North and SOPHIE observations. These new planets, WASP-52b, WASP-58b, WASP-59b, and WASP-60b, have orbital periods ranging from 1.7 to 7.9 days, masses between 0.46 and $0.94 M_{\text {Jup }}$, and radii between 0.73 and $1.49 R_{\text {Jup }}$. Their G1 to K5 dwarf host stars have $V$ magnitudes in the range 11.7-13.0. The depths of the transits are between 0.6 and $2.7 \%$, depending on the target. With their large radii, WASP-52b and WASP-58b are new cases of low-density, inflated planets, whereas WASP-59b is likely to have a large, dense core. WASP-60 shows shallow transits. In the case of WASP-52 we also detected the Rossiter-McLaughlin anomaly via time-resolved spectroscopy of a transit. We measured the sky-projected obliquity $\lambda=24_{-9}^{\circ+17}$, indicating that WASP-52b orbits in the same direction as its host star is rotating and that this prograde orbit is slightly misaligned with the stellar equator. These four new planetary systems increase our statistics on hot Jupiters and provide new targets for follow-up studies.
\end{abstract}

Key words. planetary systems - techniques: polarimetric - techniques: radial velocities

\section{Introduction}

About 200 exoplanets are known today as transiting in front of their host stars as seen from the Earth. That population is particularly interesting since it allows numerous studies, including accurate radius, mass, and density measurements, atmospheric studies in absorption through transits and in emission through occultations, dynamic analyses through possible timing variations, and obliquity measurements. Increasing the size of that sample is essential to improve our statistical knowledge on exoplanets and to discover individual cases that are particularly well-adapted to follow-up studies. Several ground-based photometric programs are surveying large fields with that goal, including SuperWASP (Pollacco et al. 2006) and HAT (Bakos et al. 2007). More recently, the dedicated space-based missions

\footnotetext{
* Radial velocities (Table 4) are only available at the CDS via anonymous ftp to cdsarc.u-strasbg. fr (130.79.128.5) or via http://cdsarc.u-strasbg.fr/viz-bin/qcat?]/A+A/549/A134
}

CoRoT (Baglin et al. 2009) and Kepler (Borucki et al. 2010) also joined that effort and have discovered new transiting exoplanets, including ones that have longer periods and smaller radii, which is not easily detectable by ground-based surveys. Because they survey smaller fields, however, CoRoT and Kepler detect planets transiting mainly in front of fainter stars, typically in the magnitude range $13<V<16$. These faint stars make follow-up observations difficult. By comparison, the planets detected by SuperWASP or HAT transit brighter stars, typically in the range $10<V<13$, allowing easier and more accurate complementary studies. Thus, ground-based surveys for detection of transiting exoplanets remain pertinent and are complementary to their space-based counterparts.

We present here the discovery of four new transiting planets detected with SuperWASP-North and SOPHIE. These two instruments allowed the detection of the first three WASP planets (Collier Cameron et al. 2007a; Pollacco et al. 2008), followed by about twenty others, including inflated hot Jupiters 
Table 1. Stellar parameters of the four planet host stars from analysis of SOPHIE spectra.

\begin{tabular}{lcccc}
\hline \hline Parameter & WASP-52 & WASP-58 & WASP-59 & WASP-60 \\
\hline RA $(J 2000)$ & $23: 13: 58.76$ & $18: 18: 48.25$ & $23: 18: 29.54$ & $23: 46: 39.98$ \\
Dec $(\mathrm{J} 2000)$ & $+08: 45: 40.6$ & $+45: 10: 19.1$ & $+24: 53: 21.4$ & $+31: 09: 21.4$ \\
$B$ & 12.9 & 12.33 & 13.92 & 12.86 \\
$V$ & 12.0 & 11.66 & 13.00 & 12.18 \\
\hline$T_{\text {eff }}(\mathrm{K})$ & $5000 \pm 100$ & $5800 \pm 150$ & $4650 \pm 150$ & $5900 \pm 100$ \\
$\log g_{*}$ & $4.5 \pm 0.1$ & $4.3 \pm 0.1$ & $4.55 \pm 0.15$ & $4.2 \pm 0.1$ \\
$\xi_{\mathrm{t}}\left(\mathrm{km} \mathrm{s}^{-1}\right)$ & $0.9 \pm 0.1$ & $1.1 \pm 0.2$ & $0.7 \pm 0.3$ & $1.0 \pm 0.1$ \\
$v_{\text {mac }}\left(\mathrm{km} \mathrm{s}^{-1}\right)$ & $0.7 \pm 0.3$ & $2.3 \pm 0.3$ & 0 & $2.9 \pm 0.3$ \\
$v \sin i_{*}\left(\mathrm{~km} \mathrm{~s}^{-1}\right)$ & $3.6 \pm 0.9$ & $2.8 \pm 0.9$ & $2.3 \pm 1.2$ & $3.4 \pm 0.8$ \\
$P_{\text {rot }}(\mathrm{d})$ & $11.8 \pm 3.3$ & $21.1 \pm 7.6$ & $15.9 \pm 8.4$ & $20.6 \pm 5.5$ \\
$\mathrm{Gyrochron} \mathrm{age}(\mathrm{Gyr})$ & $0.4_{-0.2}^{+0.3}$ & $3.2_{-2.1}^{+4.5}$ & $0.5_{-0.4}^{+0.7}$ & $3.6_{-2.1}^{+4.3}$ \\
$\log R_{\mathrm{HK}}^{\prime}$ & $-4.4 \pm 0.2$ & $-4.4 \pm 0.2$ & $-4.1 \pm 0.2$ & $-4.4 \pm 0.2$ \\
{$[\mathrm{Fe} / \mathrm{H}]$} & $0.03 \pm 0.12$ & $-0.45 \pm 0.09$ & $-0.15 \pm 0.11$ & $-0.04 \pm 0.09$ \\
{$[\mathrm{Na} / \mathrm{H}]$} & $0.29 \pm 0.08$ & $-0.44 \pm 0.07$ & - & $0.07 \pm 0.11$ \\
{$[\mathrm{Mg} / \mathrm{H}]$} & $0.26 \pm 0.13$ & $-0.30 \pm 0.07$ & - & $0.02 \pm 0.06$ \\
{$[\mathrm{Si} / \mathrm{H}]$} & $0.25 \pm 0.18$ & $-0.34 \pm 0.06$ & - & $0.15 \pm 0.08$ \\
{$[\mathrm{Ca} / \mathrm{H}]$} & $0.04 \pm 0.13$ & $-0.30 \pm 0.13$ & $0.23 \pm 0.18$ & $0.06 \pm 0.13$ \\
{$[\mathrm{Sc} / \mathrm{H}]$} & $0.17 \pm 0.11$ & $-0.22 \pm 0.07$ & - & $0.18 \pm 0.08$ \\
{$[\mathrm{Ti} / \mathrm{H}]$} & $0.06 \pm 0.12$ & $-0.29 \pm 0.07$ & $0.28 \pm 0.18$ & $0.00 \pm 0.07$ \\
{$[\mathrm{~V} / \mathrm{H}]$} & $0.19 \pm 0.13$ & - & $0.66 \pm 0.19$ & $0.02 \pm 0.10$ \\
{$[\mathrm{Cr} / \mathrm{H}]$} & $0.11 \pm 0.15$ & $-0.39 \pm 0.18$ & - & $0.05 \pm 0.14$ \\
{$[\mathrm{Co} / \mathrm{H}]$} & $0.20 \pm 0.07$ & - & $0.16 \pm 0.11$ & $-0.01 \pm 0.15$ \\
{$[\mathrm{Ni} / \mathrm{H}]$} & $0.11 \pm 0.13$ & $-0.49 \pm 0.09$ & $-0.10 \pm 0.10$ & $0.29 \pm 0.08$ \\
$\log A(\mathrm{Li})$ & $<0.3 \pm 0.2$ & $2.32 \pm 0.13$ & $<0.17 \pm 0.21$ & $<0.83 \pm 0.09$ \\
$\mathrm{Sp} . \mathrm{Type}$ & $\mathrm{K} 2 \mathrm{~V}$ & $\mathrm{G} 2 \mathrm{~V}$ & $\mathrm{~K} 5 \mathrm{~V}$ & $\mathrm{G} 1 \mathrm{~V}$ \\
$\mathrm{Distance}(\mathrm{pc})$ & $140 \pm 20$ & $300 \pm 50 \mathrm{pc}$ & $125 \pm 25$ & $400 \pm 60$ \\
\hline
\end{tabular}

such as WASP-12b (Hebb et al. 2009) and WASP-48b (Enoch et al. 2011), compact sub-hot-Jupiters such as WASP-11b (West et al. 2009), hot Jupiters on eccentric orbits such as WASP-38b (Barros et al. 2011), and low-mass giant planets, such as WASP-13b (Skillen et al. 2009) and WASP-21b (Bouchy et al. 2010).

The four new planets presented here are WASP-52b, WASP-58b, WASP-59b, and WASP-60b. They are giant, closein planets, with masses ranging from 0.46 to $0.94 M_{\text {Jup }}$, orbital periods between 1.7 and 7.9 days, and transit depths between 0.6 and $2.7 \%$. We describe in Sect. 2 the observations that allowed their discovery, detail their analyses in Sect. 3, and conclude in Sect. 4.

\section{Observations}

\subsection{Photometric detection with SuperWASP}

The coordinates and magnitudes of the stars WASP-52, 58, 59, and 60 are reported in the upper part of Table 1 . They were first identified as promising candidate host stars for transiting planets with SuperWASP-North. Located on La Palma in the Canary Islands, Spain, this facility consists of eight Canon $200 \mathrm{~mm}$ $f / 1.8$ focal lenses coupled to e $2 \mathrm{v} 2048 \times 2048$ pixel CCDs with 13.7" pixels and a field-of-view of $7.8^{\circ} \times 7.8^{\circ}$, associated with a custom-built photometric reduction pipeline (Pollacco et al. 2006). It observes with a broad-band custom filter (400-700 nm). Thousands of photometric points were secured with SuperWASP-North over several seasons for each target (see Table 2 for a summary).

Periodic signatures of possible planetary transits were identified in these light curves using the algorithms presented by Collier Cameron et al. (2006). Phase-folded SuperWASP light curves are plotted in the upper panels of Figs. 1-4, for WASP-52, 58,59 , and 60 . They show dips of about one percent, compatible
Table 2. Photometric observations.

\begin{tabular}{|c|c|c|}
\hline Telescope & Band & Date \\
\hline \multicolumn{3}{|l|}{ WASP-52 } \\
\hline SuperWASP-N & _- & 2008 Jul.-2009 Nov. \\
\hline JGT & $r$ & 2010 Oct. 24 \\
\hline FTS & $z$ & 2011 Aug. 02 \\
\hline EulerCam & $r$ & 2011 Aug. 20 \\
\hline BUSCA & $u$ & 2011 Aug. 27 \\
\hline BUSCA & $g$ & 2011 Aug. 27 \\
\hline BUSCA & $r$ & 2011 Aug. 27 \\
\hline BUSCA & $z$ & 2011 Aug. 27 \\
\hline EulerCam & $r$ & 2011 Sep. 24 \\
\hline \multicolumn{3}{|l|}{ WASP-58 } \\
\hline SuperWASP-N & _- & 2004 May-2010 Aug. \\
\hline \multicolumn{3}{|l|}{ WASP-59 } \\
\hline SuperWASP-N & - & 2004 May-2006 Nov. \\
\hline OverSky & $r$ & 2011 Oct. 28 \\
\hline OverSky & $r$ & 2011 Nov. 04 \\
\hline OverSky & $r$ & 2011 Nov. 20 \\
\hline JGT & $r$ & 2011 Nov. 20 \\
\hline \multicolumn{3}{|l|}{ WASP-60 } \\
\hline SuperWASP-N & - & 2004 May-2007 Dec. \\
\hline NITES & $r$ & 2011 Aug. 25 \\
\hline
\end{tabular}

with transiting giant planets with periods of 1.7, 5.0, 7.9, and 4.3 days.

\subsection{Radial velocities with the spectrograph SOPHIE}

The spectroscopic follow-up of these four candidates was performed with SOPHIE, the spectrograph dedicated to highprecision radial velocity measurements at the 1.93-m telescope of the Haute-Provence Observatory, France (Bouchy et al. 2009). The first goal of these observations is to establish the planetary 

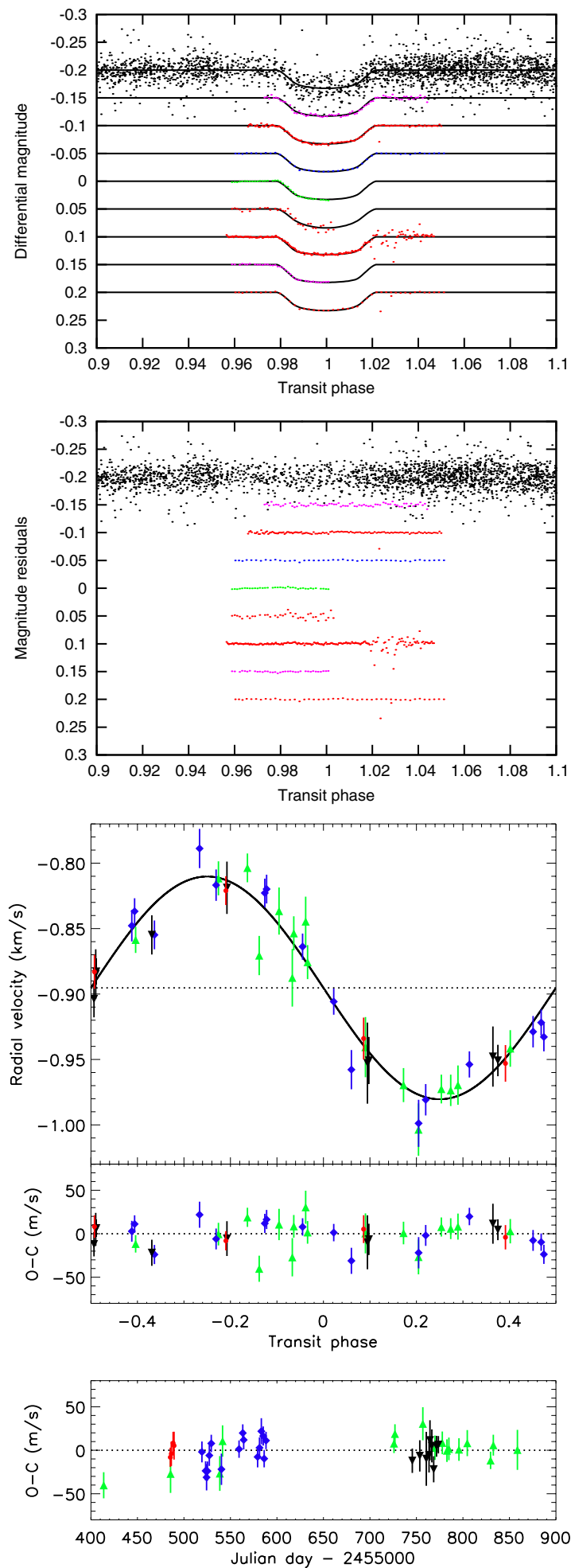

Fig. 1. Photometry and radial velocities of WASP-52. The two upper panels show the transit light curves and their residuals from the fit. Different photometric observations are ranked chronologically from top to bottom with the same order as in Table 2 . The three lower plots show the phase-folded radial velocities and their $\mathrm{O}-\mathrm{C}$ residuals from the Keplerian fit as a function of transit phase and Julian date. The data are presented as follows: SOPHIE HE1 (red circles), HE2 (blue diamonds), HR (black downward triangles), and CORALIE (green upward triangles) - see Table 4.

nature of the transiting candidates found in photometry, which in most cases are not due to planets but to astrophysical false
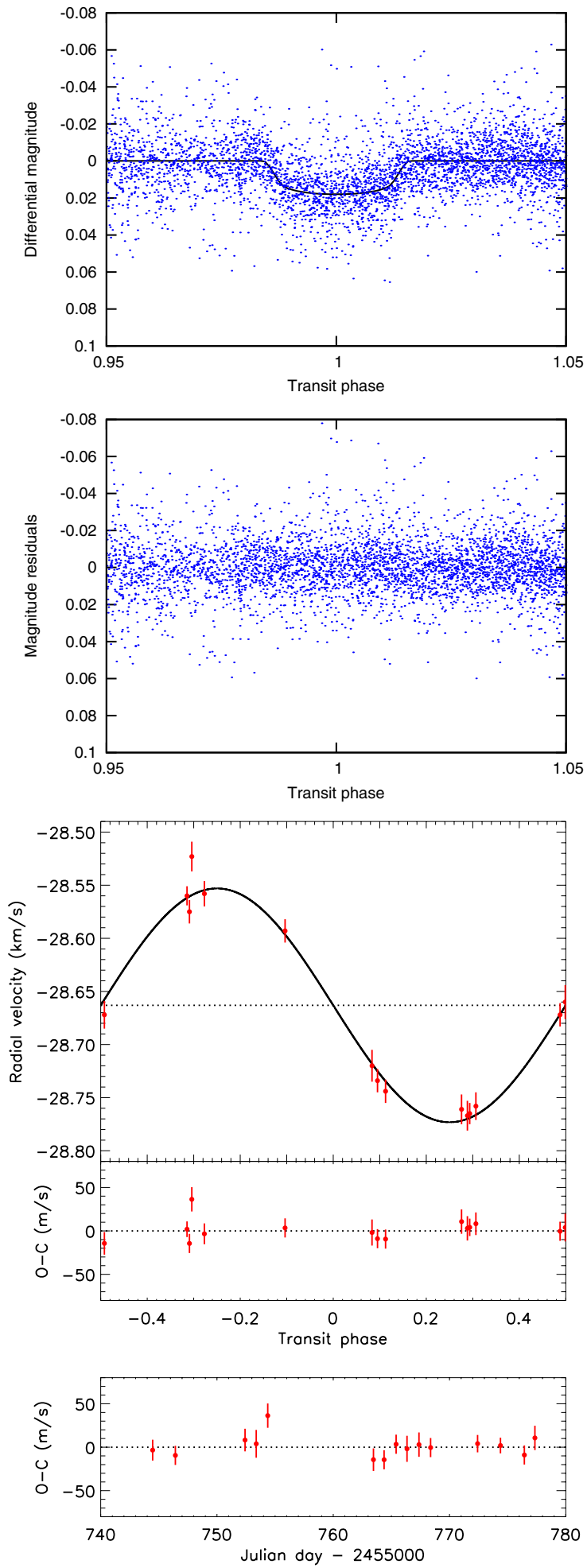

Fig. 2. Photometry and radial velocities of WASP-58. The two upper panels show the SuperWASP-N light curve and its residuals from the fit. The three lower plots show the SOPHIE radial velocities and their residuals from the Keplerian fit.

positives such as blended stellar binaries. The second goal is to characterize the secured planets by measuring in particular their masses and orbital eccentricities.

SOPHIE was mainly used in High-Efficiency mode (HE) with a resolving power $R=40000$ to increase the throughput for 

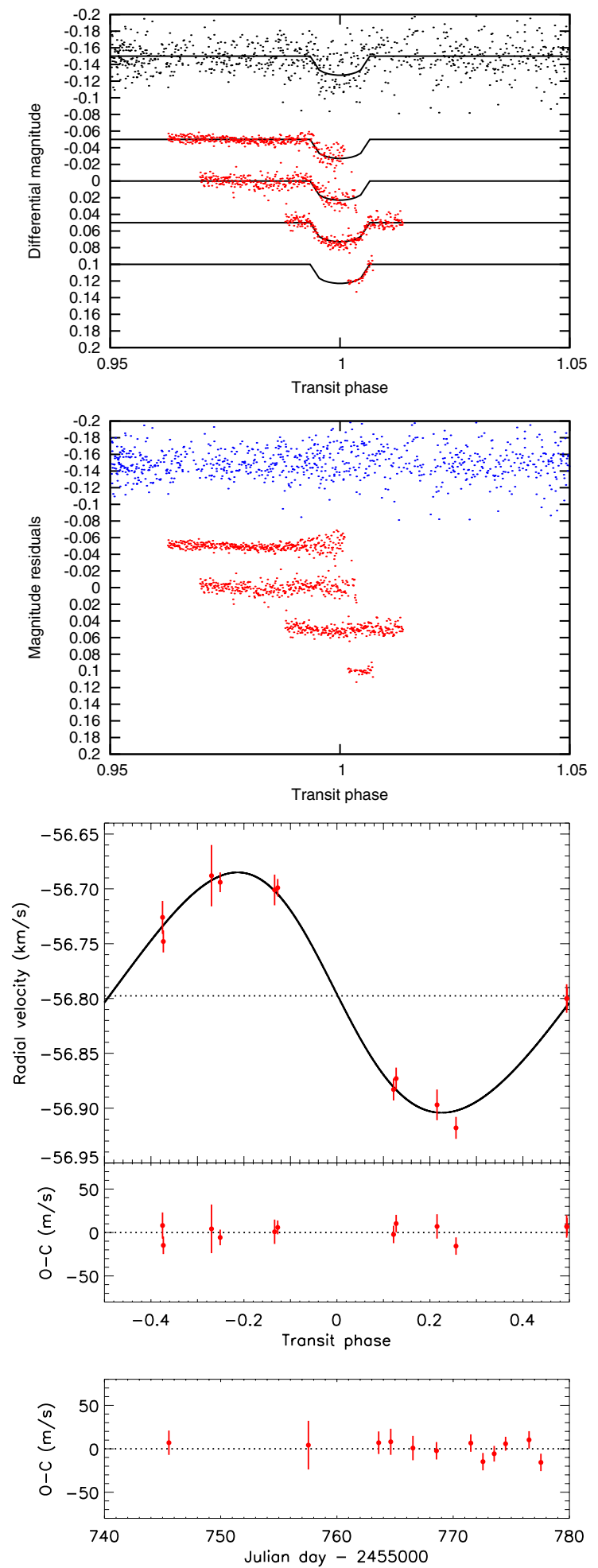

Fig. 3. Photometry and radial velocities of WASP-59. The two upper panels show the transit light curves and their residuals from the fit. Different photometric observations are ranked chronologically from top to bottom, with the same order as in Table 2. The three lower plots show the SOPHIE radial velocities and their residuals from the Keplerian fit.

these faint targets. The exposure times ranged from 10 to $30 \mathrm{~min}$ depending on the targets, and they were adjusted as a function of the weather conditions to keep the signal-to-noise ratio as constant as possible for any given target. The spectra were extracted
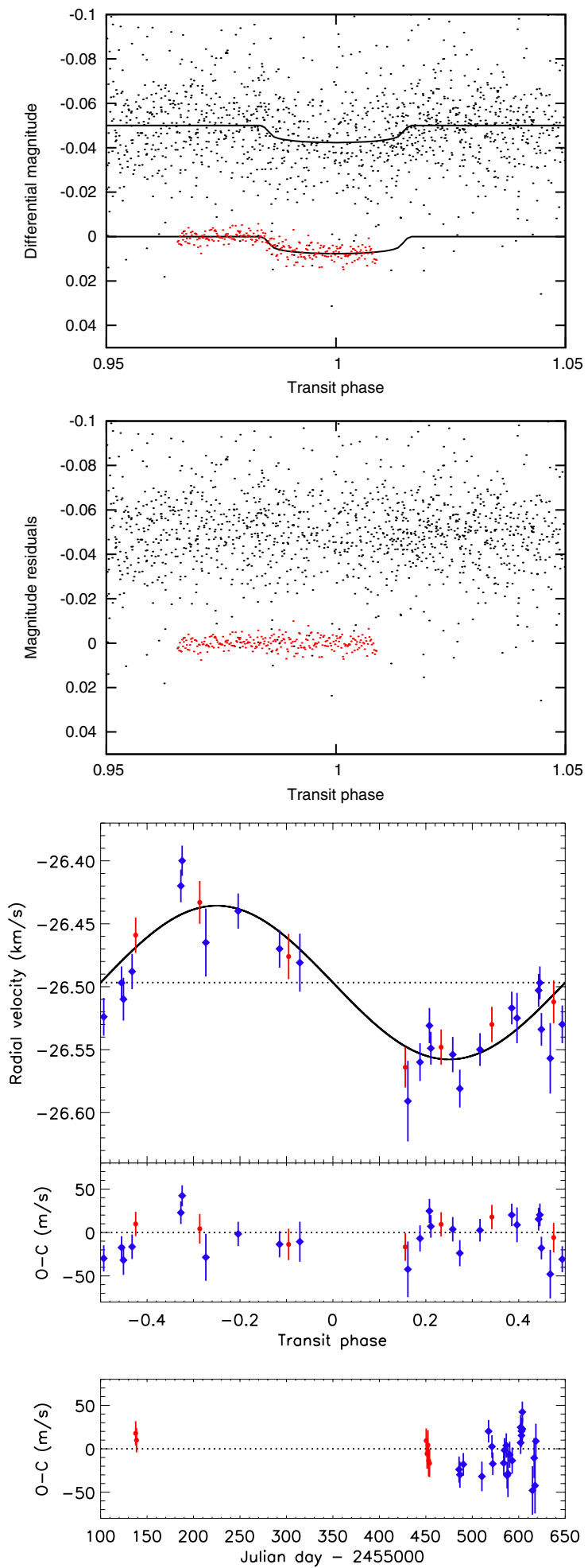

Fig. 4. Photometry and radial velocities of WASP-60. The two upper panels show the SuperWASP-N (in black) and NITES (in red) transit light curves and their residuals from the fit. The three lower plots show the SOPHIE radial velocities and their residuals from the Keplerian fit. SOPHIE HE1 and H2 (see Table 4 and Sect. 2.2) are plotted in red circles and blue diamonds, respectively.

using the SOPHIE pipeline, and the radial velocities were measured from the weighted cross-correlation with numerical masks characteristic of the spectral type of the target (Baranne et al. 1996; Pepe et al. 2002). We adjusted the number of spectral 

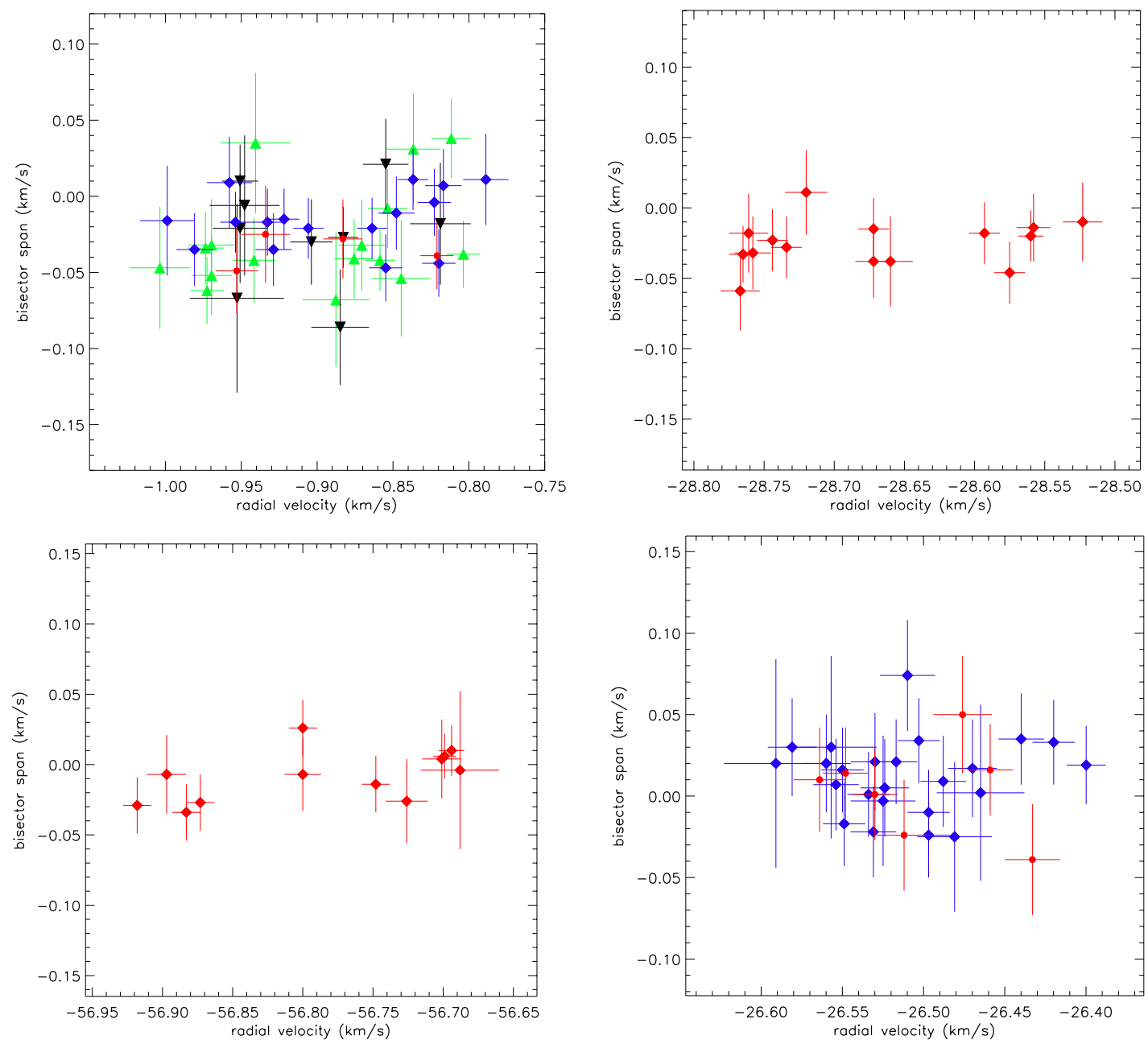

Fig. 5. Bisector span as a function of the radial velocities with 1- $\sigma$ error bars for WASP-52, 58, 59 and 60 (from left to right and top to bottom). Color codes are the same as in Figs. 1-4. The ranges here have the same extents in the $x$ - and $y$-axes.

orders used in the cross-correlation to reduce the dispersion of the measurements. Indeed, some spectral domains are noisy (especially in the blue part of the spectra), so using them degrades the accuracy of the radial-velocity measurement.

The error bars on the radial velocities were computed from the cross-correlation function using the method presented by Boisse et al. (2010). Some spectra were contaminated by moonlight. Following the method described in Pollacco et al. (2008) and Hébrard et al. (2008), we estimated and corrected for the moonlight contamination by using the second SOPHIE fiber aperture, which is targeted on the sky, while the first aperture points toward the target. This typically results in radial velocity corrections of a few $\mathrm{m} \mathrm{s}^{-1}$ up to a few tens of $\mathrm{m} \mathrm{s}^{-1}$. Removing these points does not significantly modify the orbital solutions.

The radial velocity measurements are reported in Table 4 and are displayed in the lower panels of Figs. 1-4 together with their Keplerian fits and the residuals. They show variations in phase with the SuperWASP transit ephemeris and with semiamplitudes of a few tens $\mathrm{m} \mathrm{s}^{-1}$, implying companion masses slightly below a Jupiter mass. Radial velocities measured using different stellar masks (F0, G2, or K5) produce variations with similar amplitudes, so there is no evidence that their variations could be explained by blend scenarios caused by stars of different spectral types. Similarly, the cross-correlation function bisector spans show neither variations nor trends as a function of radial velocity (Fig. 5). This reinforces the conclusion that the radial-velocity variations are not caused by spectral-line profile changes attributable to blends or stellar activity. We thus conclude that our four targets harbor transiting giant planets, which we designate as WASP-52b, WASP-58b, WASP-59b, and WASP-60b hereafter.

The raw residuals of the Keplerian fits to two of the four targets (WASP-52 and 60) show an abrupt offset of $\sim 100 \mathrm{~m} \mathrm{~s}^{-1}$ around $\mathrm{BJD}=2455500$, which could be caused by possible extra companions in the planetary systems. However, because the offset is similar in amplitude and time for both targets, this could be instrumental. WASP-58 and 59 were not observed at that epoch. To constrain possible extra companions we re-observed WASP-52 the following season with SOPHIE in High-Resolution mode (HR) with a resolving power $R=$ 75000 following the installation of new octagonal fiber sections in the spectrograph, which significantly improved the radialvelocity accuracy of SOPHIE (Bouchy et al. 2012). WASP-52 was also observed with the CORALIE spectrograph mounted on the 1.2-m Euler-Swiss telescope at La Silla (Queloz et al. 2000). These new measurements did not confirm any structure in the residuals of the one-Keplerian fit, so we conclude that the offsets detected during the previous season on WASP-52 and 60 were instrumental. Such systematic effects have already been observed in the past with the HE mode of SOPHIE, but not 
with the HR mode. They are poorly understood, but seem to be linked with temperature variations in the telescope dome. To circumvent that effect, we considered the SOPHIE HE data of WASP-52 and 60 secured before and after the zero-point change as independent datasets, allowing a free radial-velocity offset to be fitted between them. These are denoted SOPHIE HE1 and SOPHIE HE2 in Table 4.

\subsection{Additional photometry of the transits}

Once the radial velocities established that the four candidates identified with photometry indeed were transiting planets, we obtained extra transit light curves with telescopes larger than SuperWASP, allowing improved resolution and more precise time-series photometry during transit. The goal is to refine the determination of the parameters derived from photometry. It was not possible to observe extra transits of WASP-58b with our available telescopes, because the orbital period of that system is almost exactly an integer number of days $(P=5.018$ days $)$.

We used the six telescopes listed below equipped with cameras to observe additional transit light curves of the three remaining planets:

- JGT: the 0.94-m James Gregory Telescope located at University of St. Andrews, Scotland;

- FTS: the 2-m Faulkes Telescope South located at Siding Spring Observatory in New South Wales, Australia;

- EulerCam: the new camera at the 1.2-m Euler-Swiss telescope at La Silla, Chile;

- BUSCA: at the Calar Alto Observatory 2.2-m telescope, Spain;

- OverSky: the 0.36-m telescope at the OverSky Observatory, La Palma, Spain;

- NITES: a 0.4-m telescope at La Palma, Spain.

The dates of the transit observations made with these facilities as well as the filters used are reported in Table 2. Standard aperture photometry was carried out on all these datasets, using comparison stars available in the different fields for relative photometry. The extra light curves of WASP-52, 59, and 60 are plotted in the upper panels of Figs. 1, 3, and 4.

\subsection{Transit spectroscopy}

A transit of WASP-52b was observed in spectroscopy with SOPHIE. The goal was to detect the Rossiter-McLaughlin anomaly, which is an apparent time-dependent distortion of the stellar line profiles that occurs when a planet transits the disk of a rotating star. It allows the measurement of the sky-projected angle between the planetary orbital axis and the stellar rotation axis, usually denoted $\lambda$ (see, e.g., Hébrard et al. 2008). The spectroscopic transit was observed in the HE mode of SOPHIE to improve the throughput.

The target was observed continuously during a four-hour sequence under good weather conditions on 2011 August 21. Nineteen measurements were secured with exposure times ranging from 600 to $1200 \mathrm{~s}$, including nine within the transit. The remaining observations obtained before and after the transit are mandatory for reference points. The radial velocities were extracted as above (Sect. 2.2), but using slightly fewer orders for the cross-correlation to reduce the dispersion of the measurements. This depends on the flux color balance in the spectra, which in turn depends on the airmasses of the different observations.
These observations are plotted in Fig. 8 and are labeled SOPHIE HE-RM in Table 4. The Rossiter-McLaughlin anomaly is detected, with a full amplitude of $\sim 80 \mathrm{~m} \mathrm{~s}^{-1}$. The shape of the anomaly indicates a prograde orbit, with an asymmetry that suggests misalignment (see Sect. 3.3).

For different reasons, including poor weather conditions, we were unable to perform time-resolved spectroscopy of the transits of the three other planets.

\section{Analysis}

\subsection{Parameters of the host stars}

Spectral analysis of the four host stars was performed with the co-added individual spectra obtained with SOPHIE for radial velocity measurements (Sect. 2.2). We used here only the spectra without moonlight contamination and only the HE spectra out of transit in the case of WASP-52. It enables a sufficiency high signal-to-noise ratio to be reached with a given spectral resolution, and avoid any possible contamination in the spectra. The parameters obtained from these analyses are listed in the bottom part of Table 1.

We used the methods described by Gillon et al. (2009). The $\mathrm{H}_{\alpha}$ line was used to determine the effective temperature $T_{\mathrm{eff}}$, except for WASP-59 where $\mathrm{H}_{\alpha}$ was too weak; for that star $T_{\text {eff }}$ was measured from the null dependence of abundance on excitation potential. The surface gravity $\log g_{*}$ was determined from the Ca I lines at $6162 \AA$ and $6439 \AA$ (Bruntt et al. 2010a), along with the $\mathrm{Na} I \mathrm{D}$ and $\mathrm{Mg}$ I $\mathrm{b}$ lines. The spectral types were established from $T_{\text {eff }}$ using the table in Gray (2008).

The elemental abundances were determined from equivalent width measurements of several clean and unblended lines. The microturbulence $\xi_{\mathrm{t}}$ was evaluated from Fe I using the method of Magain (1984). The quoted error estimates include that given by the uncertainties in $T_{\text {eff }}, \log g_{*}$ and $\xi_{\mathrm{t}}$, as well as the scatter due to measurement and atomic data uncertainties.

The projected stellar rotation velocities $v \sin i_{*}$ were determined by fitting the profiles of several unblended Fe I lines. They agree with the measurements secured from the crosscorrelation function following Boisse et al. (2010). The values for macroturbulence $v_{\mathrm{mac}}$ were assumed based on the tabulations by Gray (2008) and Bruntt et al. (2010b). Macroturbulence was assumed to be zero for WASP-59, since for mid-K stars it is expected to be lower than that of thermal broadening (Gray 2008). An instrumental FWHM of $0.15 \pm 0.01 \AA$ was measured from the telluric lines around $6300 \AA$, and best-fitting values of the $v \sin i_{*}$ were obtained.

Lithium is detected only in WASP-58. The temperature of $5800 \mathrm{~K}$ along with the derived lithium abundance $\log A(\mathrm{Li}) \mathrm{im}-$ ply a lower age limit of $\sim 2$ Gyr when compared with NGC 752 (Sestito \& Randich 2005). There is no significant detection of lithium in the spectra of WASP-52, 59 and 60, with equivalent width upper limits of $4 \mathrm{~m} \AA, 15 \mathrm{~m} \AA$, and $6 \mathrm{~m} \AA$. The lack of lithium would suggest lower age limits of $0.5 \mathrm{~Gy}$ and several Gyr for WASP-52 and 60 (Sestito \& Randich 2005). For the mid-K star WASP-59, lithium is expected to be depleted in only a few 100 Myr.

For WASP-52, 58, 59, and 60, the rotation rates implied by the $v \sin i_{*}\left(P_{\text {rot }}=11.8 \pm 3.3 \mathrm{~d}, 21.1 \pm 7.6 \mathrm{~d}, 15.9 \pm 8.4 \mathrm{~d}\right.$, and $20.6 \pm 5.5 \mathrm{~d})$ give gyrochronological ages of $0.4_{-0.2}^{+0.3} \mathrm{Gyr}$, $3.2_{-2.1}^{+4.5} \mathrm{Gyr}, 0.5_{-0.4}^{+0.7} \mathrm{Gyr}$, and 3.6 $6_{-2.1}^{+4.3}$ Gyr using the Barnes (2007) relation.

WASP-52 and 59 show chromospheric emission peaks in the $\mathrm{Ca}$ II $\mathrm{H}+\mathrm{K}$ lines that indicate they are active stars, whereas 

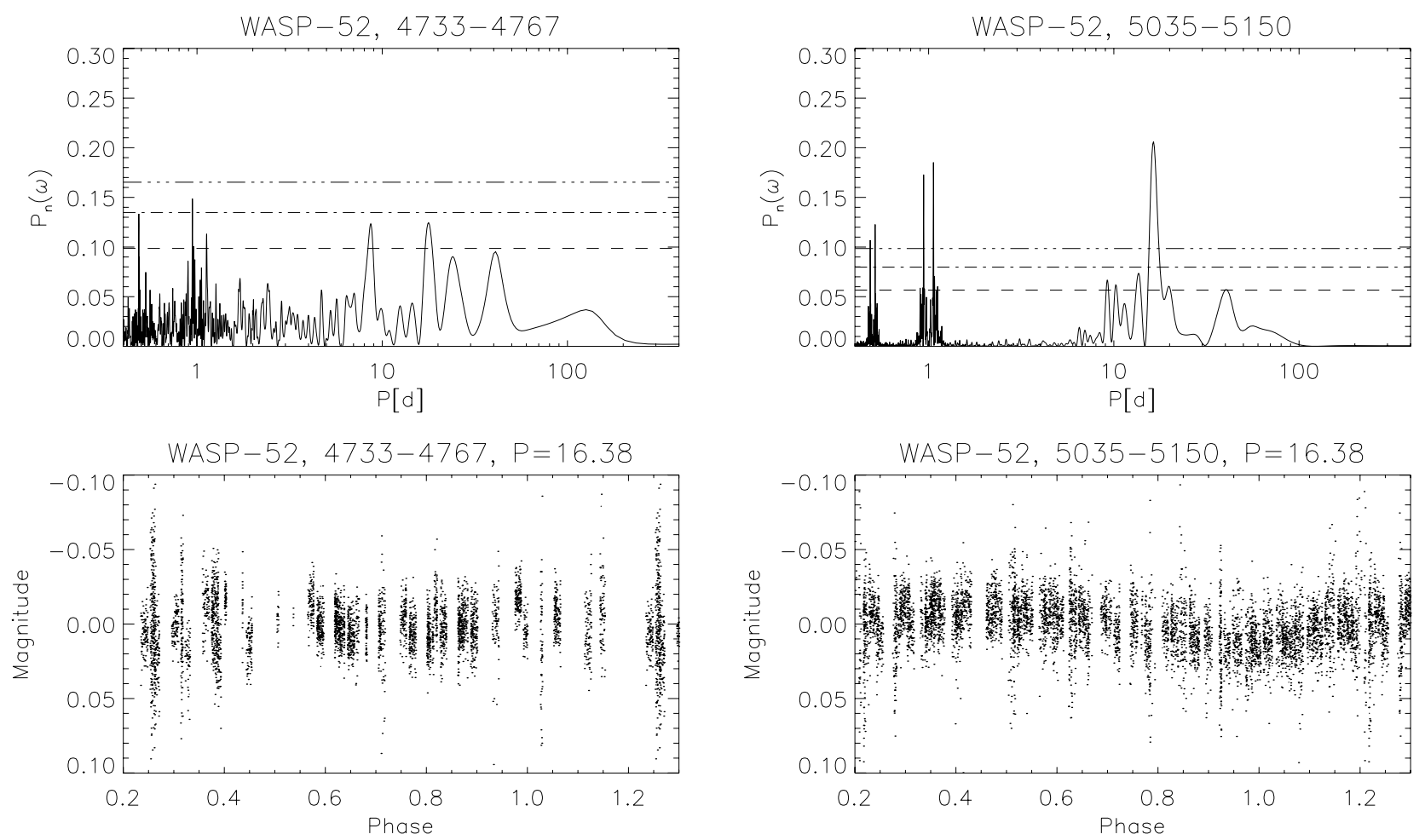

Fig. 6. Upper panel: periodograms for the WASP data from two seasons for WASP-52. The date range (JD-245000) is given in the title of each panel. Horizontal lines indicate false-alarm probability levels $\mathrm{FAP}=0.1,0.01,0.001$. Lower panel: data for the two seasons folded on the period $P=16.38$ days.

WASP-58 and 60 do not show any evident emission. We computed the $\log R_{\mathrm{HK}}^{\prime}$ indices following the calibration of Boisse et al. (2010)

The SuperWASP light curve of the active star WASP-52 shows long-term modulations. EulerCam data also show irregularities. We have analyzed the SuperWASP light curve to determine whether they are periodic, and possibly due to the combination of magnetic activity and the rotation of the star. The value of $v \sin i_{*}$ derived above together with the estimated stellar radius implies a rotation period of 5-15 days, assuming that the rotation axis of the star is approximately aligned with the orbital axis of the planet. We show below in Sect. 3.3 that this is not exactly the case, but the misalignment remains low. We used the sine-wave fitting method described in Maxted et al. (2011) to calculate the periodograms shown in Fig. 6. These are calculated over 4096 uniformly spaced frequencies from 0 to $2.5 \mathrm{cy}-$ cles/day. The false-alarm probability levels shown in these figures are calculated using a boot strap Monte Carlo method also described in Maxted et al. (2011). Variability due to star spots is not expected to be coherent on long timescales as a consequence of the finite lifetime of star-spots and differential rotation in the photosphere, and so we analyzed the two seasons of data for WASP-52 separately.

The second season of data shows a highly significant periodic modulation with a period of $16.4 \pm 0.04$ days and an amplitude of 9.6 milli-magnitudes. We used a boot strap Monte Carlo method to estimate the error on this period. We also calculated periodograms of three stars with similar magnitudes and colors to WASP-52 observed simultaneously with the same WASP camera. None of these stars showed significant periodic modulation in the same frequency interval. The strongest peak in the first season of data is marginally significant and is consistent with the one-day alias of the period detected in the second season of data.

\subsection{Parameters of the planetary systems}

\subsubsection{Method}

For a given system, all available data were fitted simultaneously: the radial velocities with a Keplerian orbit, and the transit light curves with the Mandel \& Agol (2002) algorithm using four non linear limb-darkening coefficients from the tabulations of Claret (2004). The global fit was made with the latest version of the Markov-chain Monte Carlo (MCMC) procedure presented in detail by Collier Cameron et al. (2007b) and Pollacco et al. (2008). The free parameters in the fits are the transit epoch $T_{0}$, the orbital period $P$, the depth of the transit or the planet/star area ratio $\left(R_{\mathrm{p}} / R_{*}\right)^{2}$, the total transit duration $t_{\mathrm{T}}$, the impact parameter $b$, the eccentricity $e$ of the orbit, the argument of periastron $\omega$, the semi-amplitude $K_{1}$ of the radial velocity variations, the $n$ systemic radial velocities $\gamma_{n}$ (one for each of the $n$ radial velocity datasets). When no significant eccentricity is detected (in three out of the four systems), the orbits are considered as circular in the final fit. The stellar density $\rho_{*}$ is directly derived from the transit light curve (through $P, t_{\mathrm{T}}$, and $b$ values). The density, together with $T_{\text {eff }}$ and $[\mathrm{Fe} / \mathrm{H}]$, yields the stellar mass $M_{*}$ and radius $R_{*}$ through empirical calibrations established for eclipsing binaries (Torres et al. 2010; Enoch et al. 2011). From these we conclude that the four host targets are dwarf stars. The planetary mass $M_{\mathrm{p}}$ and radius $R_{\mathrm{p}}$ are derived from $K_{1}$ and $\left(R_{\mathrm{p}} / R_{*}\right)^{2}$, which directly provides the planetary density $\rho_{\mathrm{p}}$. We also computed the derived parameters as the star surface gravity $\log g_{*}$ (which here is more accurate than that obtained from spectral analysis), the semi-major axis $a$ and the orbital inclination $i_{\mathrm{p}}$ as well as the equilibrium temperature $T_{\mathrm{P}}$ of the planet, assuming it to be a black body and that energy is efficiently redistributed from the planets day-side to its night-side. 

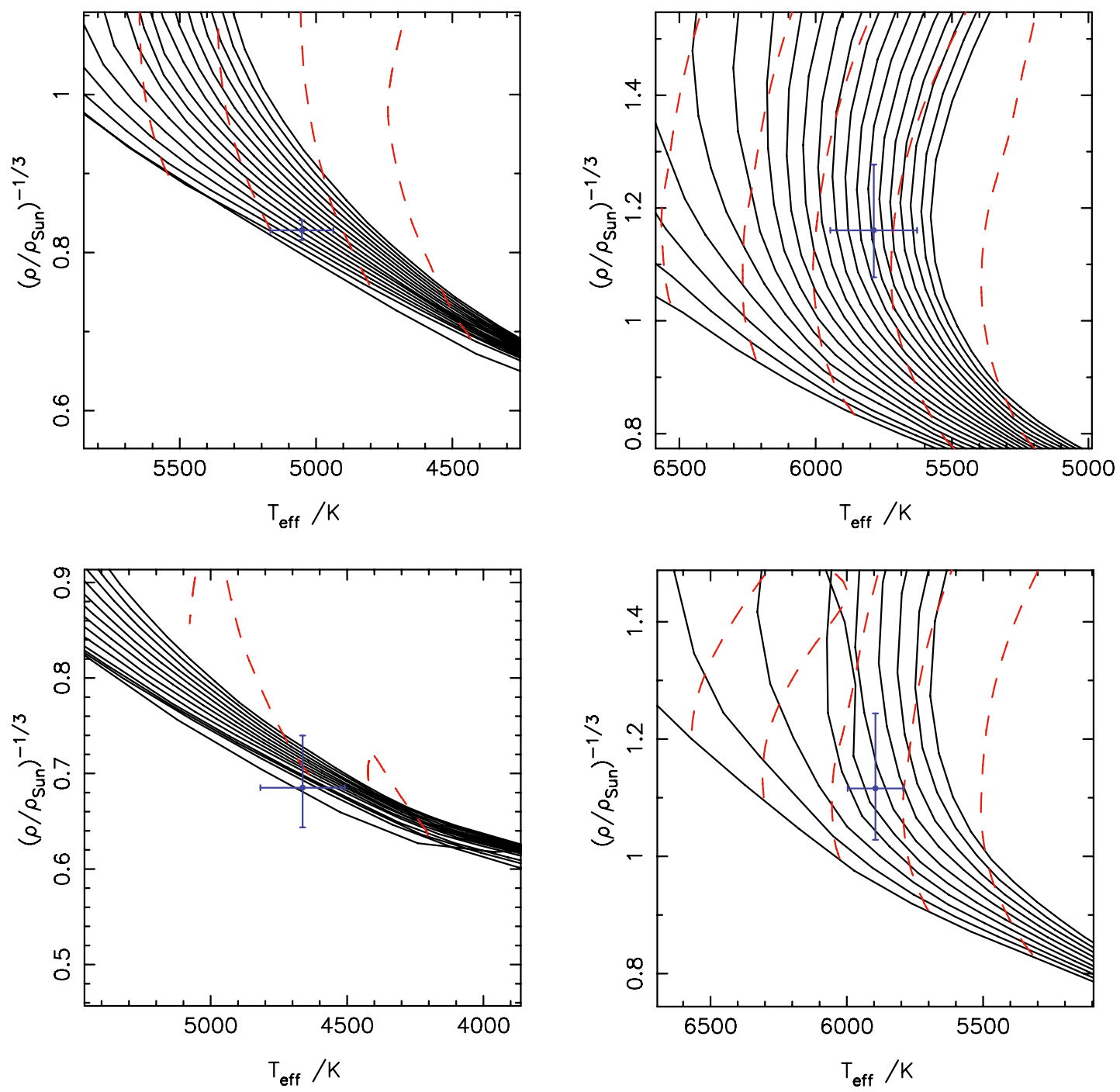

Fig. 7. HR diagrams for WASP-52, 58, 59, and 60 (Yonsei-Yale stellar models). Each host star is denoted by a blue cross as a function of the stellar density and the effective temperature. Solid, black lines are the isochrones, and red, dashed lines are the evolutioanry tracks for different masses. WASP-52 (upper, left): $0.1 \mathrm{Gyr}$ isochrone, and then $1 \mathrm{Gyr}$ to $15 \mathrm{Gyr}$ in $1 \mathrm{Gyr}$ increments, and mass tracks from 1.0 to $0.7 M_{\odot}$ in 0.1 increments from left to right. WASP-58 (upper, right): 1 to $20 \mathrm{Gyr}$ in $1 \mathrm{Gyr}$ increment isochrones, and mass tracks from 1.1 to $0.7 M_{\odot}$ in 0.1 increments from left to right. WASP-59 (lower, left): $0.1,0.4,0.6 \mathrm{Gyr}$ isochrones, then $1 \mathrm{Gyr}$ to $10 \mathrm{Gyr}$ in $1 \mathrm{Gyr}$ increments, and 0.7 and $0.6 M_{\odot}$ mass tracks from left to right. WASP-60 (lower, right): $1 \mathrm{Gyr}$ to $10 \mathrm{Gyr}$ in $1 \mathrm{Gyr}$ increments, and mass tracks from 1.3 to $0.70 M_{\odot}$ in 0.1 increments from left to right.

For comparison, the stellar mass was also derived from mass tracks in $\rho_{*}$-vs. $-T_{\text {eff }}$ HR diagrams (Sozzetti et al. 2007). We compared several models for the tracks in the HR diagrams: the Padova isochrones of Marigo et al. (2010), the Teramo stellar models from Pietrinferni et al. (2004), the Victoria-Regina stellar models from Vandenberg et al. (2006), and the Yonsei-Yale isochrones of Demarque et al. (2004). The last among these are plotted in Fig. 7 as examples. We also estimated the stellar ages from the isochrones in these HR diagrams; although their uncertainties are on the order of several Gyr, they remain in broad agreement with gyrochronological ages.

The best fits of the WASP-52, 58, 59, and 60 systems are plotted over the data in Figs. 1-4. The median values and 1- $\sigma$ uncertainties of the parameters obtained from the MCMC are reported in Table 3. Each system is detailed below.

\subsubsection{WASP-52}

The stellar mass obtained from the MCMC and that estimated from mass tracks in evolution diagrams for WASP-52 agree well.
The red noise levels in the light curves are negligible; averaging the photometric data in 6- to 10 -min bins made no appreciable difference to the error bars on the derived parameters. The radial velocity fit provides systemic radial velocities of $-0.8953 \pm 0.0075,-1.0415 \pm 0.0033,-0.9265 \pm 0.0062$, and $-0.8877 \pm 0.0040 \mathrm{~km} \mathrm{~s}^{-1}$ for SOPHIE HE1, HE2, HR, and CORALIE, respectively, with a dispersion of the residuals of about $13 \mathrm{~m} \mathrm{~s}^{-1}$. We fit the data using only parts of the radial velocity datasets, which provided similar results. This suggests that the systematic shift of $146 \pm 8 \mathrm{~m} \mathrm{~s}^{-1}$ between the two SOPHIE HE datasets (see Sect. 2.2) has no significant impact on the derived parameters of the system. There is a hint of a drift of $\sim+40 \mathrm{~m} \mathrm{~s}^{-1}$ over the 15 -month time span of our observations. It could be caused by an extra component in the system, but the unknown systematic shifts between datasets do not allow any firm conclusion to be drawn on the source of the drift. No significant eccentricity is detected.

The orbital period is 1.75 day. The one-day alias of that signal is 2.33 days; it should be investigated because the photometric and radial velocity observations were made with a typical 
Table 3. Planetary and stellar parameters for the systems WASP-52, 58, 59, and 60.

\begin{tabular}{|c|c|c|c|c|}
\hline Parameter & Symbol & WASP-52 & WASP-58 & Units \\
\hline Transit epoch (HJD-2 450 000.0) & $T_{0}$ & $5793.68143 \pm 0.00009$ & $5183.9335 \pm 0.0010$ & days \\
\hline Orbital period & $P$ & $1.7497798 \pm 0.0000012$ & $5.017180 \pm 0.000011$ & days \\
\hline Transit duration & $t_{\mathrm{T}}$ & $0.0754 \pm 0.0005$ & $0.1582 \pm 0.0043$ & days \\
\hline Planet/star area ratio & $\left(R_{\mathrm{p}} / R_{*}\right)^{2}$ & $0.0271 \pm 0.0004$ & $0.0145 \pm 0.0010$ & \\
\hline Impact parameter & $b$ & $0.60 \pm 0.02$ & $0.46 \pm 0.23$ & $R_{*}$ \\
\hline Scaled stellar radius & $R_{*} / a$ & $0.1355 \pm 0.0020$ & $0.097 \pm 0.011$ & \\
\hline Stellar density & $\rho_{*}$ & $1.76 \pm 0.08$ & $0.58 \pm 0.19$ & $\rho_{\odot}$ \\
\hline Stellar surface gravity & $\log g_{*}$ & $4.582 \pm 0.014$ & $4.27 \pm 0.09$ & (cgs) \\
\hline Stellar radius & $R_{*}$ & $0.79 \pm 0.02$ & $1.17 \pm 0.13$ & $R_{\odot}$ \\
\hline Stellar mass & $M_{*}$ & $0.87 \pm 0.03$ & $0.94 \pm 0.10$ & $M_{\odot}$ \\
\hline Semi-major axis & $a$ & $0.0272 \pm 0.0003$ & $0.0561 \pm 0.0020$ & $\mathrm{AU}$ \\
\hline Orbital inclination & $i_{\mathrm{p}}$ & $85.35 \pm 0.20$ & $87.4 \pm 1.5$ & $\circ$ \\
\hline Stellar reflex velocity & $K_{1}$ & $0.0843 \pm 0.0030$ & $0.1101 \pm 0.0041$ & $\mathrm{kms} \mathrm{s}^{-1}$ \\
\hline Orbital eccentricity & $e^{1}$ & 0 (fixed) & 0 (fixed) & \\
\hline Planet radius & $R_{\mathrm{p}}$ & $1.27 \pm 0.03$ & $1.37 \pm 0.20$ & $R_{\mathrm{J}}$ \\
\hline Planet mass & $M_{\mathrm{p}}$ & $0.46 \pm 0.02$ & $0.89 \pm 0.07$ & $M_{\mathrm{J}}$ \\
\hline Planet surface gravity & $\log g_{\mathrm{p}}$ & $2.81 \pm 0.03$ & $3.03 \pm 0.12$ & (cgs) \\
\hline Planet density & $\rho_{\mathrm{p}}$ & $0.22 \pm 0.02$ & $0.34 \pm 0.14$ & $\rho_{\mathrm{J}}$ \\
\hline Planetary equilibrium temperature & $T_{\mathrm{P}}$ & $1315 \pm 35$ & $1270 \pm 80$ & $\mathrm{~K}$ \\
\hline Parameter & Symbol & WASP-59 & WASP-60 & Units \\
\hline Transit epoch (HJD-2 450 000.0) & $T_{0}$ & $5830.95559 \pm 0.00053$ & $5747.0295 \pm 0.0022$ & days \\
\hline Orbital period & $P$ & $7.919585 \pm 0.000010$ & $4.3050011 \pm 0.0000062$ & days \\
\hline Transit duration & $t_{\mathrm{T}}$ & $0.102 \pm 0.002$ & $0.139 \pm 0.005$ & days \\
\hline Planet/star area ratio & $\left(R_{\mathrm{p}} / R_{*}\right)^{2}$ & $0.0169 \pm 0.0008$ & $0.0060 \pm 0.0006$ & \\
\hline Impact parameter & $b$ & $0.29 \pm 0.18$ & $0.37 \pm 0.23$ & $R_{*}$ \\
\hline Scaled stellar radius & $R_{*} / a$ & $0.041 \pm 0.003$ & $0.100 \pm 0.011$ & \\
\hline Stellar density & $\rho_{*}$ & $3.11 \pm 0.64$ & $0.72 \pm 0.20$ & $\rho_{\odot}$ \\
\hline Stellar surface gravity & $\log g_{*}$ & $4.72 \pm 0.06$ & $4.35 \pm 0.09$ & $(\mathrm{cgs})$ \\
\hline Stellar radius & $R_{*}$ & $0.613 \pm 0.044$ & $1.14 \pm 0.13$ & $R_{\odot}$ \\
\hline Stellar mass & $M_{*}$ & $0.719 \pm 0.035$ & $1.078 \pm 0.035$ & $M_{\odot}$ \\
\hline Semi-major axis & $a$ & $0.0697 \pm 0.0011$ & $0.0531 \pm 0.0006$ & AU \\
\hline Orbital inclination & $i_{\mathrm{p}}$ & $89.27 \pm 0.52$ & $87.9 \pm 1.6$ & $\circ$ \\
\hline Stellar reflex velocity & $K_{1}$ & $0.1096 \pm 0.0044$ & $0.0608 \pm 0.0038$ & $\mathrm{kms} \mathrm{s}^{-1}$ \\
\hline Orbital eccentricity & $e$ & $0.100 \pm 0.042$ & 0 (fixed) & \\
\hline Argument of periastron & $\omega$ & $74 \pm 15$ & - & $\circ$ \\
\hline Planet radius & $R_{\mathrm{p}}$ & $0.775 \pm 0.068$ & $0.86 \pm 0.12$ & $R_{\mathrm{J}}$ \\
\hline Planet mass & $M_{\mathrm{p}}$ & $0.863 \pm 0.045$ & $0.514 \pm 0.034$ & $M_{\mathrm{J}}$ \\
\hline Planet surface gravity & $\log g_{\mathrm{p}}$ & $3.52 \pm 0.08$ & $3.19 \pm 0.12$ & (cgs) \\
\hline Planet density & $\rho_{\mathrm{p}}$ & $1.8 \pm 0.5$ & $0.8 \pm 0.3$ & $\rho_{\mathrm{J}}$ \\
\hline Planetary equilibrium temperature & $T_{\mathrm{P}}$ & $670 \pm 35$ & $1320 \pm 75$ & $\mathrm{~K}$ \\
\hline
\end{tabular}

one-day frequency. The periodograms show a signal at both periods (1.75 and 2.33 days). However, the radial velocity data folded at 2.33 days clearly provide a poorer fit, with a dispersion of $30 \mathrm{~m} \mathrm{~s}^{-1}$ in the residuals, more than twice higher than the 1.75-day solution. This confirms that 1.75 day is the correct orbital period of the planet.

WASP-52b is a hot Jupiter that has less than half the mass of Jupiter, but a significantly larger radius. It is thus a new example of an inflated hot Jupiter. Together with the small radius of its $\mathrm{K} 2 \mathrm{~V}$ host star, this makes the planetary transits particularly deep at $2.7 \%$. The case of WASP-52b should act as a warning, since many transit signals of this high depth detected by photometric surveys may be flagged as caused by binaries, and may be a priori removed from radial-velocity follow-up (see also Southworth et al. 2012). Hence, there may be an underlying population of heavily inflated planets with large transit depths that have been mistakenly identified as binaries and hence their true nature has never been revealed.

\subsubsection{WASP-58}

WASP-58 has larger uncertainties on its derived parameters because no extra transit light curves were obtained in complement to the original SuperWASP data. Assuming a main-sequence mass-radius prior would reduce the uncertainties, in particular on the stellar and planetary radii, but we did not make that assumption in our final results to remain conservative. This does not change the parameters significantly.

For that target, the empirical estimation of the stellar mass derived from $T_{\text {eff }}$ and $[\mathrm{Fe} / \mathrm{H}]$ (Torres et al. 2010; Enoch et al. 2011) provides $M_{*}=1.03 \pm 0.05 M_{\odot}$, whereas the isochrones favor lower values around $M_{*}=0.82 \pm 0.06 M_{\odot}$. This discrepancy may be due to the low metallicity of the star, which could be beyond the validity of the Torres-Enoch calibration. Metal-poor main-sequence stars of a given mass do indeed burn hotter than their metal-rich counterparts. The metallicity of WASP-58 has to be increased to at least -0.2 to allow a better agreement between the two estimates. However, when we consider other cases where hot Jupiters orbit low-metallicity host stars (e.g. WASP-21, 37, or 78) we do not find similar discrepancies between the two estimates. Thus we remain uncertain of the true source of the disagreement. To reflect this, we have conservatively adopted an intermediate value for the stellar mass with an increased uncertainty to $M_{*}=0.94 \pm 0.10 M_{\odot}$.

The radial velocities have a systemic value of $-28.6631 \pm$ $0.0033 \mathrm{~km} \mathrm{~s}^{-1}$ and their residuals show a $11-\mathrm{m} \mathrm{s}^{-1}$ dispersion, 
in agreement with the expected uncertainties on their measurements. No structure or drift are seen in the residuals, providing no evidence of any extra component in the system.

Regardless of whether we assume a mass-radius relationship for the host star, we find that WASP-58b is a typical inflated hot Jupiter, with a radius 1.4 times that of Jupiter, and a similar mass. Its orbital period of 5.0 days is located in the higher tail of the hot Jupiter period distribution. It orbits a particularly metalpoor star, whereas most hot Jupiters preferentially orbit metalrich stars.

\subsubsection{WASP -59}

The systemic radial velocity of WASP-59 is $-56.7976 \pm$ $0.0043 \mathrm{~km} \mathrm{~s}^{-1}$ with a $10-\mathrm{m} \mathrm{s}^{-1}$ dispersion around the circular Keplerian fit and no detection of any additional drift. The stellar mass estimate from the MCMC analysis agrees with that derived from evolutionary tracks in the density-temperature plane. As expected for late K-type stars, the isochrones are too closely spaced to allow them to be distinguished, and gyrochronology provides the only age estimate. By allowing the eccentricity to vary freely, we found $e=0.10 \pm 0.04$ and a dispersion of the radial velocity residuals decreased to $5 \mathrm{~m} \mathrm{~s}^{-1}$. If the marginal $(2 \sigma)$ detection of an eccentric orbit is confirmed, then it may be in part due to the 7.9-day orbital period, which is relatively long compared to typical hot Jupiters. Indeed, tidal circularization is less effective at larger orbital separations, and initial eccentricities in the planetary orbit may not yet have been damped (e.g. Hut 1981). Following Matsumura et al. (2008), we estimated the circularization time to be $5 \mathrm{Gyr}$, which is about ten times longer than the gyrochronological age.

WASP-59b is one of the rare hot Jupiters found by groundbased transit searches with orbital periods longer than seven days, together with WASP-8b, HAT-P-15b, and HAT-P-17b. Detecting such transiting objects from ground-based photometry from a single site remains difficult because of the night/day duty cycle. The space-based missions CoRoT and Kepler provide continuous photometry, and are therefore more suited to detect planets with longer orbital periods such as CoRoT-4b, 6b, 10b, 20b, and Kepler-28c, 29b, 29c, 32c.

The $0.7-R_{\text {Jup }}$ radius of WASP-59b places it just between the hot Jupiters (with radii typically between 1.0 and $1.4 R_{\text {Jup }}$ and periods shorter than ten days) and the small-radius planets recently detected mainly by Kepler (with radii typically between 0.1 and $\left.0.5 R_{\mathrm{Jup}}\right)$. Only a few objects are known in that intermediate radius range. One of the few others is the planet HD 149026b (Sato et al. 2005), whose density indicates a large dense core. Interestingly enough, the planet WASP-59b also has a particularly high density. Its host star is also one of the rare stars cooler than $5000 \mathrm{~K}$ that harbor a hot Jupiter.

\subsubsection{WASP-60}

The systemic radial velocities of WASP-60 are $-26.4967 \pm$ $0.0103 \mathrm{~km} \mathrm{~s}^{-1}$ and $-26.6288 \pm 0.0054 \mathrm{~km} \mathrm{~s}^{-1}$ for the two HE datasets. The systematic shift between them $\left(132 \pm 12 \mathrm{~m} \mathrm{~s}^{-1}\right)$ agrees with that found above for WASP-52 $\left(146 \pm 8 \mathrm{~m} \mathrm{~s}^{-1}\right)$; this reinforces our conclusion that this shift has an instrumental origin (see Sect. 2.2). The radial velocity residuals show a $20-\mathrm{m} \mathrm{s}^{-1}$ dispersion, in agreement with the expected error bars on their measurements. There is a hint of a drift of $\sim+70 \mathrm{~m} \mathrm{~s}^{-1}$ over the 16-month time span of our observations. Again, this may suggest the presence of an additional body in the system, but the unknown systematic shift between the two datasets prevents any firm conclusion from being drawn.
The stellar mass obtained from the MCMC analysis agrees with that estimated from evolutionary tracks in the densitytemperature plane diagrams for WASP-60. The planet appears to have a Saturn-like radius and sub-Jupiter mass, making it an interestingly compact for a planet orbiting a slightly metalpoor star.

\subsection{Rossiter-McLaughlin anomaly analysis of WASP-52}

The radial velocities of WASP-52 measured during the 2011 August 21 transit were fitted to derive the sky-projected angle $\lambda$ between the planetary orbital axis and the stellar rotation axis. We applied the analytical approach developed by Ohta et al. (2005) to model the form of the Rossiter-McLaughlin anomaly, which is described by ten parameters: the stellar limb-darkening linear coefficient $\epsilon$, the transit parameters $R_{\mathrm{p}} / R_{*}, a / R_{*}$ and $i_{\mathrm{p}}$, the parameters of the circular orbit $\left(P, T_{0}\right.$, and $\left.K_{1}\right)$, the systemic radial velocity, and finally $v \sin i_{*}$ and $\lambda$. We adopted $\epsilon=0.82$ computed by Claret (2004) for the $g^{\prime}$ filter corresponding to the SOPHIE wavelength range. The transit and orbital parameters were determined above from the light curves and radial velocity fits. Their uncertainties are negligible here for the fit of the Rossiter-McLaughlin anomaly. We checked that varying them in their 1- $\sigma$ intervals has no impact on the quality of the fit or on the derived $\lambda$ value. The main parameters that play a role in this fit are the systemic velocity of that particular dataset, $\lambda$, and $v \sin i_{*}$. As these parameters could be correlated in the Rossiter-McLaughlin fit, we computed the $\chi^{2}$ of the fit on a three-dimensional grid for which we scanned all their possible values.

The systemic velocity was determined with a $\pm 5-\mathrm{m} \mathrm{s}^{-1}$ accuracy. It is constrained thanks to the observations secured immediately before and after the transit. The confidence interval contours estimated from $\chi^{2}$ variations for the $\lambda$ and $v \sin i_{\text {* }}$ show no correlation between these two parameters, as expected for systems whose impact parameter $b$ is significantly different from zero. From $\chi^{2}$ variations, we obtained $\lambda=24_{-9}^{\circ+17}$ and $v \sin i_{*}=2.5 \pm 1.0 \mathrm{~km} \mathrm{~s}^{-1}$. The best fit is plotted in Fig. 8 .

The dispersion of the residuals about the fit is $20 \mathrm{~m} \mathrm{~s}^{-1}$, in agreement with the expected uncertainties. The Rossiter-McLaughlin anomaly detection is noisy and only spans a few points. As a statistical test for the detection, we computed the $\chi^{2}$ over the measurements secured during the transit night for the fits first including the Rossiter-Mclaughlin anomaly, and then compared this to the fits achieved when the RM anomaly is ignored. The F-test indicates that the $\chi^{2}$ improvement with the Rossiter-McLaughlin fit has a probability $>65 \%$ to be indeed caused by the Rossiter-McLaughlin anomaly detection. We therefore concluded that the spectroscopic transit is significantly detected.

Owing to the quality of the the data, we remain cautious with respect to the numerical result on $\lambda$. The orbit of WASP-52b is apparently prograde and slightly misaligned. We note that the $v \sin i_{*}$ value obtained from the fit agrees with the value obtained from spectral analysis (see Table 1).

\section{Conclusion}

We have presented the discovery of four new transiting exoplanets, WASP-52b, WASP-58b, WASP-59b, and WASP-60b, detected mainly from SuperWASP-North and SOPHIE observations. As with most of the transiting planets found from groundbased observatories, they can be ranked in the hot-Jupiter class. Each of them has some peculiarities, however, WASP-52b and WASP-58b are new cases of inflated planets, increasing the 


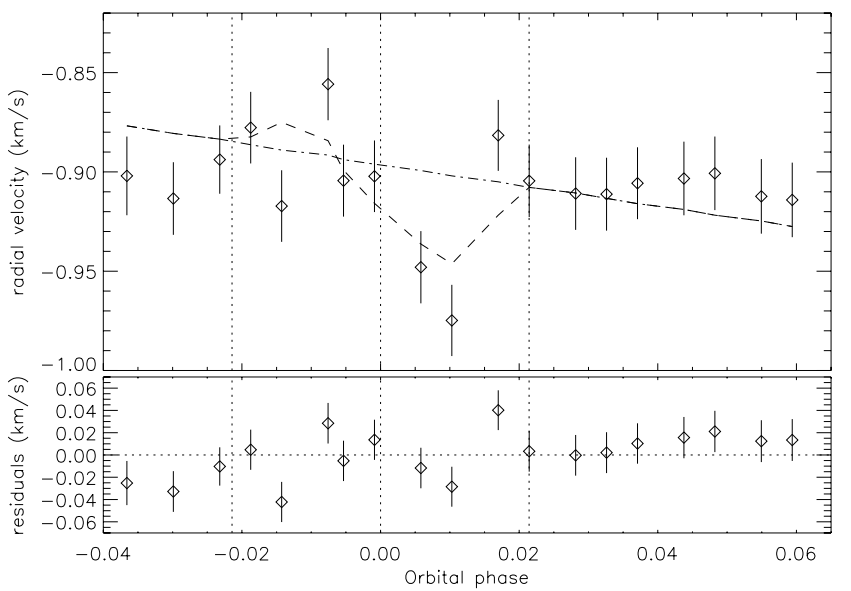

Fig. 8. Spectroscopic observation of the transit of WASP-52b. Top: SOPHIE radial velocity measurements as a function of the orbital phase (diamonds), Keplerian fit ignoring the transit (dotted-dashed line), and final fit including the model of the Rossiter-McLaughlin anomaly (dashed line). The vertical dotted lines show the times of mid-transit, first, and fourth contacts. Bottom: residuals of the final fit.

population of giant planets with abnormally large radii whose origin is not well understood yet despite the numerous models proposed to explain them (see, e.g., Fortney \& Nettelmann 2010). WASP-52 is a new case of a possibly slightly misaligned system; it does not fit with the possible correlations of $\lambda$ with $T_{\text {eff }}$ (Winn et al. 2010) or $M_{\mathrm{p}}$ (Hébrard et al. 2011), however. The hot Jupiter WASP-58b orbits a particularly metal-poor star. WASP$59 \mathrm{~b}$ has an orbital period longer than typical transiting hot Jupiters and a high density, suggesting a large core. Finally, WASP-60b presents shallow transits of $0.6 \%$, illustrating the capabilities of ground-based surveys to detect small-size planets. These new planets provide new targets for follow-up studies and increase our statistics on well-characterized exoplanets, allowing progress to be made in the comprehension of the formation and evolution of planetary systems.

Acknowledgements. We thank the 2010 cohort of the AS4025 Observational Astrophysics class at the University of St. Andrews for their help in analyzing and eliminating astrophysical false positives in the JGT data, and for recognizing WASP-52 as a good potential planet candidate. The research leading to these results has received funding from the European Communitys Seventh Framework Programme (FP7/2007-2013) under grant agreement number RG226604 (OPTICON), as well as the Programme National de Planétologie (PNP) of CNRS/INSU (France). RFD is supported by CNES.

\section{References}

Baglin, A., Auvergne, M., Barge, P., et al. 2009, Transiting Planets, Proc. IAU Symp., 253, 71

Bakos, G. À., Noyes, R. W., Kovàcs, G., et al. 2007, ApJ, 656, 552

Baranne, A., Queloz, D., Mayor, M., et al. 1996, A\&AS, 119, 373

Barnes, S. A. 2007, ApJ, 669, 1167

Barros, S. C. C., Faedi, F., Collier Cameron, A., et al. 2011, A\&A, 525, A54

Boisse, I., Eggenberger, A., Santos, N. C., et al. 2010, A\&A, 523, A88

Borucki, W. J., Koch, D. G., Basri, G., et al. 2010, Science, 327, 977

Bouchy, F., Hébrard, G., Udry, S., et al. 2009, A\&A, 505, 853

Bouchy, F., Hebb, L., Skillen, I., et al. 2010, A\&A, 519, A98

Bouchy, F., Díaz, R. F., Hébrard, G., et al. 2012, A\&A, 549, A49

Bruntt, H., Deleuil, M., Fridlund, M., et al. 2010a, A\&A, 519, A51

Bruntt, H., Bedding, T. R., Quirion, P.-O., et al. 2010b, MNRAS, 405, 1907

Claret, A. 2004, A\&A, 428, 1001

Collier Cameron, A., Pollacco, D., Street, R. A., et al. 2006, MNRAS, 373, 799

Collier Cameron, A., Bouchy, F., Hébrard, G., et al. 2007a, MNRAS, 375, 951

Collier Cameron, A., Wilson, D. M., West, R. G., et al. 2007b, MNRAS, 380, 1230

Demarque, P., Woo, J.-H. K., Yong-Cheol, Y., \& Sukyoung K. 2004, ApJS, 155, 667

Enoch, B., Collier Cameron, A., Parley, N. R., \& Hebb, L. 2010, A\&A, 516, A33

Enoch, B., Anderson, D. R., Barros, S., et al. 2011, ApJ, 142, 86

Fortney, J. J., \& Nettelmann, N. 2010, Space Sci. Rev., 152, 423

Gillon, M., Smalley, B., Hebb, L., et al. 2009, A\&A, 496, 259

Gray, D. F. 2008, The observation and analysis of stellar photospheres, 3rd edn. (Cambridge University Press), 507

Hebb, L., Collier-Cameron, A., Loeillet, B., et al. 2009, MNRAS, 393, 1920

Hébrard, G., Bouchy, F., Pont, F., et al. 2008, A\&A, 481, 52

Hébrard, G., Ehrenreich, D., Bouchy, F., et al. 2011, A\&A, 527, L11

Hut, P. 1981, A\&A, 99, 126

Magain, P. 1984, A\&A, 134, 189

Mandel, K., \& Agol, E. 2002, ApJ, 580, L171

Marigo, P., Girardi, L., Bressan, A., et al. 2010, A\&A, 482, 883

Matsumura, S., Takeda, G., \& Rasio, F. 2008, ApJ, 686, L29

Maxted, P. F. L., Anderson, D. R., Collier Cameron, A., et al. 2011, PASP, 123, 547

Ohta, Y., Taruya, A., \& Suto, Y. 2005, ApJ, 622, 1118

Pepe, F., Mayor, M., Galland, F., et al. 2002, A\&A, 388, 632

Pietrinferni, A., Cassisi, S., Salaris, M., \& Castelli, F. 2004, ApJ, 612, 168

Pollacco, D. L., Skillen, I., Collier Cameron, A., et al. 2006, PASP, 118, 140

Pollacco, D., Skillen, I., Collier Cameron, A., et al. 2008, MNRAS, 385, 1576

Queloz, D., Mayor, M., Weber, L., et al. 2000, A\&A, 354, 99

Sato, B., Fischer, D. A., Henry, G. W., et al. 2005, ApJ, 633, 465

Sestito, P., \& Randich, S. 2005, A\&A, 442, 615

Skillen, I., Pollacco, D., Collier Cameron, A., et al. 2009, A\&A, 502, 391

Southworth, J., Hinse, T. C., Dominik, M., et al. 2012, MNRAS, 426, 1338

Sozzetti, A., Torres, G., Charbonneau, D., et al. 2007, ApJ, 664, 1190

Torres, G., Andersen, J., \& Giménez, A. 2010, A\&ARv, 18, 67

VandenBerg, D. A., Bergbusch, P. A., \& Dowler, P. D. 2006, ApJS, 162, 375

West, R. G., Collier Cameron, A., Hebb, L., et al. 2009, A\&A, 502, 395

Winn, J. N., Fabrycky, D., Albrecht, S., \& Johnson, J. A. 2010, ApJ, 718, L145 